$\mathbb{T}$ periodica polytechnica

\author{
Transportation Engineering \\ $38 / 1(2010) 45+51$ \\ doi: 10.3311/pp.tr.2010-1.08 \\ web: http://www.pp.bme.hu/tr \\ (c) Periodica Polytechnica 2010
}

RESEARCH ARTICLE

\section{Vehicle modeling for integrated control design}

Balázs Németh / Péter Gáspár

Received 2009-09-03

\begin{abstract}
This paper comprises the control-oriented modeling of an integrated vehicle system including powertrain, brake, active suspension and steering components. As the first step, the models of a vehicle are formalized. In the powertrain model the nonlinearities of an engine with thermodynamical and fluid mechanical features are considered. In the model of suspension the dynamics of sprung mass (body) and unsprung masses (e.g., wheels) are considered. In the brake system the necessary hydraulic pressure required for the desired brake power is determined. The pressure fluctuation arising from the skewness of brake disc during the rotation is distributed. The mechanical construction of electronic steering system is also dealt with. The model of the vehicle handles the effects of road and wind disturbances. Matlab/Simulink, CarSim and GT-Suite GT-Power are used during the simulations. Matlab/Simulink is a perfect tool for control design and a fast analysis of the operation of the controlled system. CarSim is a widely used vehicle dynamic simulator used wide-spread; GT-Power is a complex powertrain modeling program. Both are used for validating Matlab models.
\end{abstract}

\section{Keywords}

control-oriented modeling $\cdot$ powertrain system $\cdot$ steering system $\cdot$ brake system $\cdot$ active suspension $\cdot$ optimal control

\section{Acknowledgement}

The research was supported by the Hungarian National Science Foundation (OTKA) through grant CNK-78168 "Modelling and multi-objective optimization based control of road traffic flow considering social and economical aspects" which is gratefully acknowledged.

\section{Balázs Németh}

Department of Control and Transport Automation, BME, Bertalan L. u. 2. H1111 Budapest, Hungary

e-mail: nemeth.balazs@mail.bme.hu

\section{Péter Gáspár}

Systems and Control Laboratory, Computer and Automation Research Institute, MTA, Kende u. 13-17, H-1111 Budapest, Hungary

e-mail: gaspar@sztaki.hu

\section{Introduction and motivation}

The significant increase in the number of automotive electrical components in automobiles has initiated a revolution in the car industry. For this change to happen the development of electrical and digital computing sciences, the general use of integrated circuits and the low cost and reliability of these components were necessary. The different control systems of the vehicle have one common control unit which controls the systems to be harmonized. It means not only a micro-controller, but also a control algorithm, which is able to control alone several systems belonging to it. Another advantage of using integrated systems is the changeability of the functions. For example if the steering system fails, the vehicle will stay controllable by the moment from the difference between brake forces acting on the wheels. In the integrated control the Electronic Stability Program (ESP), Anti-Blocking System (ABS), Anti-Slip Regulation (ASR), active steering, active suspension, electronic powertrain may be used. Nowadays integrated control is only in the research phase because of the failures of control theories and because the existing systems are not fully-fledged for standardized production.

For control design it is necessary to construct a model, in which the dynamics of vehicle is taken into consideration. The finite element method results in a high-complexity model, which can be used for analysis purposes, e.g. [6]. However, for numerical reasons the model which is the basis of the control design must be of reduced order. The features which are important in terms of the controlled system are selected and the other features are ignored. The purpose of the paper is to construct a model which contains the powertrain, steering, suspension and brake systems. Several methods have been elaborated to formalize vehicle and tyre models, see e.g. [1, 3, 5, 7].

The purpose of the modeling is the design of a controller, thus the model of vehicle systems must be augmented with performance specifications and model uncertainties.

This paper is organized as follows: Section 2 contains the models of vehicle systems, such as the engine, brake, suspension and steering; this section shows their control-oriented application. The constructed models are validated in Section 3. and the last section summarizes the achievements. 


\section{Control-oriented vehicle model}

\subsection{Engine model}

There are several possibilities for modeling an engine, and they differ in terms of methods, the complexity of the models used and also the time required for the solutions. An engine model contains thermodynamical and fluid mechanical differential-equations; the mechanical processes can be modeled by dynamic equations [4,5].

The model formalizes the intake/exhaust manifold by using equations of continuity, Navier-Stokes equation and the conservation of energy. There are also algebraic equations, which describe pipe friction, pressure-loss, heat transfer, pipe connections etc. The model of the fuel injection is based on a periodically and momentary injected fuel

$$
\sigma_{f}=\alpha A_{i n j} \sqrt{2 \rho_{f}\left(p_{i n j}-p_{c y l}\right)}
$$

The volume of the doses is $m_{d}=\eta_{v o l} \rho_{\text {ref }} V_{D} \frac{1}{\lambda K_{\text {stoch }}}$.

In simple cases thermodynamical procedures are modeled by two-zone thermodynamical models. The model must be built from the conservation of mass and energy. The cylinder cycles are modeled by the empiric Viebe-burning model according to reaction-kinetics [4]. The cumulative speed of burning is

$$
x=\frac{m_{f}}{m_{f s u m}}=1-e^{-a y^{m+1}},
$$

where $y$ is the relative burning duration

$$
y=\frac{\varphi-\varphi_{\text {start }}}{\varphi_{\text {end }}-\varphi_{\text {start }}}
$$

$m$ is the Viebe-form parameter and $a$ is the speed parameter.

The law of Woschni describes the heat transfer of the piston, cylinder wall, cylinder head, which are heat abstractions in the cylinder cycle. This equation is also based on experience, therefore the choice of parameters is crucial. The quantity of lead heat-rate is:

$$
\frac{d Q_{w}}{d t}=k_{1} A_{1}\left(T-T_{w 1}\right)+k_{2} A_{2}\left(T-T_{w 2}\right)+k_{3} A_{3}\left(T-T_{w 3}\right) .
$$

The description of gas-transfer processes is possible by using the conservation of mass. The mass-flow of gas in the cylinder is equal to the difference in the mass-flow of the intake and the exhaust gas: $d m_{x}=d m_{i n t}-d m_{e x h}$. The energy change of gas in the cylinder can be described by the conservation of energy. Here the first law of thermodynamics is used [2]:

$$
d\left(m_{x} u_{x}\right)=d m_{i n t} u_{i n t}-d m_{e x h} u_{e x h}-d Q_{f} \pm p_{x} d V_{x}
$$

The differential-equations are the following:

$$
\begin{aligned}
& \frac{d p_{x}}{d t}=p_{x}(A-B-C-D) \\
& \frac{d T_{x}}{d t}=T_{x}\left[\left(1-\frac{T_{x}}{\kappa_{i n t} T_{i n t}}\right) A-\frac{\kappa_{x}-1}{\kappa_{x}}(B+C)-D\right]
\end{aligned}
$$

where A,B,C,D are complex equations, they are found in [2].
The description of the cam throttle control is possible with the valve-lift by using a look-up-table if the valve-lift description is difficult in an implicit form. In case of general tasks valve lifting can be described by using harmonic cam profiles: before the inflexion point $h_{\varphi}=b(1-\cos \varphi)$, after the inflexion point $h_{\beta}=h_{B}-b_{1}[1-\cos (\alpha-\phi)]$; where $b$ is the difference between basic circle radius and the cam radius, $h_{B}$ is the maximal lift, and $b_{1}$ is the distance between the center of the basic circle and the cam radius.

The rotation of the crankshaft can be described by kinematical equations of the engine, like the conservation of impulse:

$$
\frac{d\left(\Theta_{e} \omega_{e}\right)}{d t}=M_{\text {ind }}-M_{\text {fric }}-M_{\text {load }}
$$

and $\frac{\varphi}{t}=\omega_{e}$

The indicated moment of the engine (frictional and drive losses of auxiliary machines are added to the effective moment) is $M_{i n d}=\sum p A_{D} \frac{\sin \left(\varphi_{e}-\psi_{e}\right)}{\cos \left(\psi_{e}\right)} r$. The friction moment is $M_{\text {fric }}=\frac{p_{\text {fric }} V_{D}}{i \phi}$, where the mean pressure is $p_{\text {fric }}=$ $A+B p_{\text {max }}+C \cdot c_{k}+D \cdot c_{k}^{2}$.

Modeling cylinder cycle of an engine is a complex and complicated exercise. GT-Suite GT-Power is an engine-modeling software, which contains non-linear thermodynamical, fluid and mechanical equations of internal-combustion engines. Further modeling and control design are performed in Matlab/Simulink, in which the achievements of GT-Power simulation are used to set engine parameters.

- Intake manifold system: Using the described fluid mechanical equations, the engine model could be rather complex and its solution is difficult. Since the model is only used for control design some simplification must be carried out. Instead of using this solution, the characteristics (look-up tables) of intake manifold system from GT-Power are used.

- Injection system: The engine is assumed to work in stoichiometric air ratio and this condition does not change significantly.

- Engine cylinder cycle: The engine model in Matlab uses equations with the suitable Viebe form and burning-speed parameters. When the gas transfer, burning and continuity are described the equations between the opening of the exhaust valves and the closing of the intake valves can be solved. In the burning phase of the cylinder cycle the Viebe equation is used.

- Cam throttle control: The engine model in this paper is a 16valve (2-camshaft) overhead-controlled DOHC. Valve lifting is defined by harmonic cam profile, which approximates the suitable reality and could be handled easily mathematically.

In terms of the control design the output of the controller is a traction force, which must be transformed into throttle position. First, torque is computed from the desired driving force 
calculated by the controller

$$
M_{\text {control }}=\frac{F_{\text {control }}}{k_{\text {gear }} \cdot k_{\text {axleratio }} / r_{g}} .
$$

In the second step, the throttle position must be read from the rev-throttle-torque characteristics of the engine.

\subsection{Automatic transmission and differential gear model}

The automatic transmission has several advantages in practice, because in this way gear-shifting can be a part of the integrated control. The required shift is determined by an electronic control unit using several considerations.

The modeled transmission in this paper is a six-stage automatic transmission, similar to ZF 6 HP 26. Its epicyclic unit has two main parts, an elemental epicyclic gear and a Ravigneaux gear (Fig. 1). These two parts are connected by clutches $(\mathrm{A}, \mathrm{B}, \mathrm{E})$. In order to realize several ratios brakes $(\mathrm{C}, \mathrm{D})$ are also necessary to reduce the degrees of freedoms. The model of transmission is built up in Matlab, using the SimMechanics blockset. The friction coefficients of brakes and clutches depend on the rotational speed of lamellas (slip). The torque capacity of the clutch is as follows [8]:

$$
T_{c i}=A_{i} \mu_{i} P_{i} \operatorname{sgn}\left(c_{i, s l i p}\right),
$$

where $A_{i}$ is the area of clutch, $\mu_{i}$ is the friction coefficient, $P_{i}$ is the clutch pressure and $c_{i, \text { slip }}$ is the clutch slip.

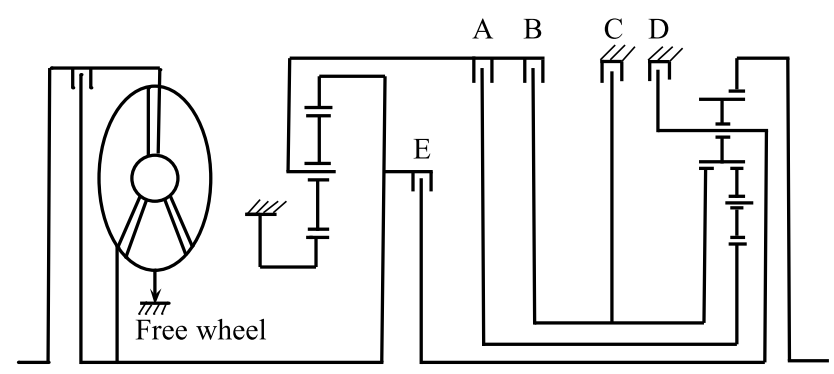

Fig. 1. Mechanic structure of ZF 6 HP 26

Considering that in the integrated control design the torque conversation of the transmission has the most important role, in control-orientated modeling only this property is taken into consideration. Therefore in the control design they are only multipliers representing torque conversation. The torque ratios of the shifted levels are: 3.5 - 2.2 - 1.3 - 1.1 - 0.85 - 0.59; driven axle ratio is 4.1 .

\subsection{Braking model}

Nowadays the most important control functions of brake systems are ABS and ASR. An elementary model of ABS/ASR control consists of a wheel, actual torque (traction or brake) and the traction/brake force on wheel-road contact (Fig. 2).

The equations of the controlled wheel are:

$$
\begin{aligned}
& \theta_{w} \ddot{\phi}=M_{w}-F_{\text {long }} R \\
& s=\frac{v-v_{k}}{v}=\frac{v-\omega R}{v}
\end{aligned}
$$

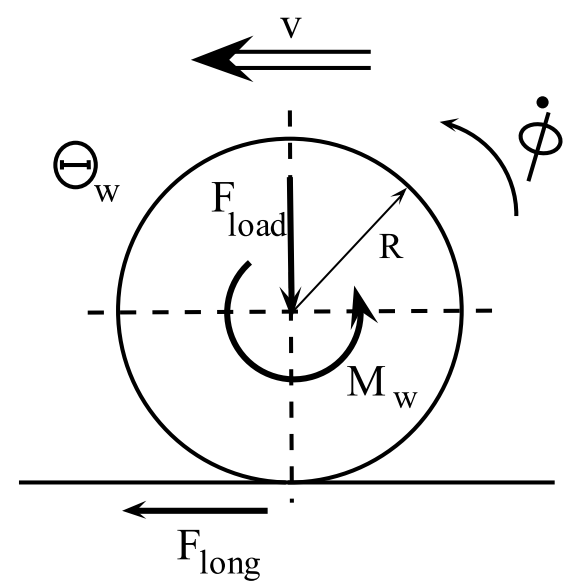

Fig. 2. Elementary model for ABS/ASR control

where $s$ is the slip of wheel, $v$ is the velocity, $\dot{\phi}$ is the rotational speed of the wheel, $R$ is the wheel radius, $\theta$ is the inertia of the wheel, $F_{\text {long }}$ is the longitudinal (tractive or braking) force and $M_{w}$ is control torque.

A purpose of ABS/ASR control design is to influence the wheel longitudinal slip. It is a very complicated automotive problem, because the slip depends on a wide range of parameters. In order to reduce a possible skidding the goal is to exploit the maximal adhesion coefficient.

Several kinds of tasks are realized by using wheels. Wheels can transmit tractive/braking forces, by differential braking it is possible to realize a torque effect on the vehicle. At a twolevel control hierarchy an upper-level controller computes the necessary yaw-torque and longitudinal force of the vehicle. The lower-level controller distributes the forces between the wheels and transforms these values for the physical input of the actuator.

Using the equations of the ABS control (9) and (10) the model can be transformed into the state-space representation form:

$$
\left[\begin{array}{l}
\dot{\phi} \\
\ddot{\phi}
\end{array}\right]=\left[\begin{array}{ll}
0 & 1 \\
0 & 0
\end{array}\right]\left[\begin{array}{l}
\phi \\
\dot{\phi}
\end{array}\right]+\left[\begin{array}{c}
0 \\
\frac{1}{\theta_{w}}
\end{array}\right] M_{w}+\left[\begin{array}{c}
0 \\
-\frac{R}{\theta_{w}}
\end{array}\right] F_{\text {long }}
$$

Supposing that the optimal longitudinal slip value according to the maximal tractive/braking force is 0.2 , the Eq. (10) can be transformed using $0.8 v-v_{k}=1$. Thus the force, which can be realized on the wheel-road contact is $F_{\text {load }}=\mu F_{\text {long }}$, where $\mu$ is the adhesion coefficient.

The purpose of the control design is to achieve a calculated slip. The state-space representation is

$$
\begin{aligned}
{\left[\begin{array}{c}
\dot{\phi} \\
\ddot{\phi} \\
\dot{y_{s l}}
\end{array}\right]=} & {\left[\begin{array}{ccc}
0 & 1 & 0 \\
0 & 0 & 0 \\
-1 & 0 & 0
\end{array}\right]\left[\begin{array}{c}
\phi \\
\dot{\phi} \\
y_{s l}
\end{array}\right]+\left[\begin{array}{c}
0 \\
\frac{1}{\theta_{w}} \\
0
\end{array}\right] M_{w}+} \\
+ & {\left[\begin{array}{c}
0 \\
-\frac{R}{\theta_{w}} \\
0
\end{array}\right] F_{\text {long }}+\left[\begin{array}{l}
0 \\
0 \\
1
\end{array}\right] \text { slip }_{\text {ref }} }
\end{aligned}
$$

The equations of the linear quadratic ABS/ASR show that the reference value of the longitudinal slip must be calculated and 

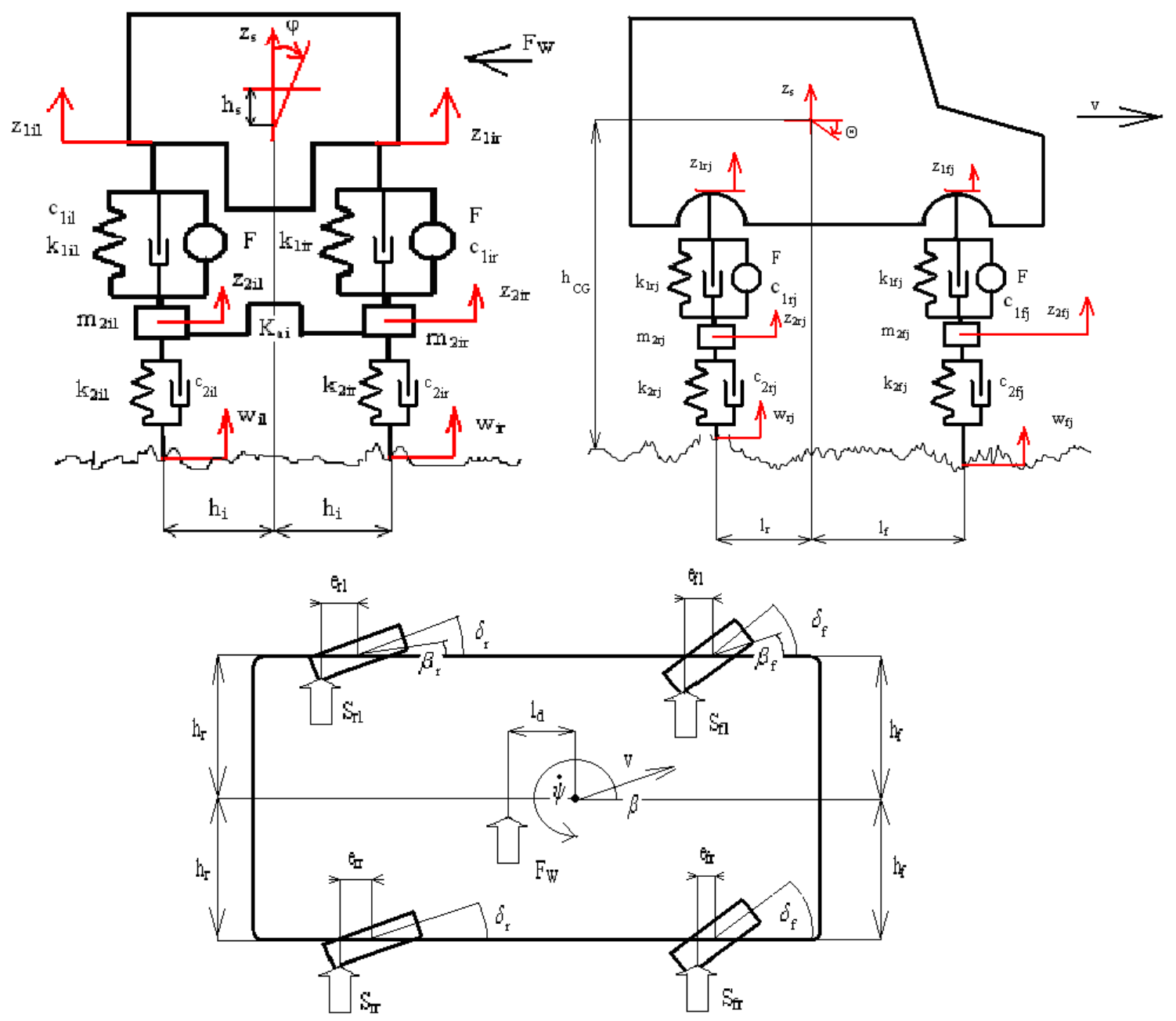

Fig. 3. Suspension model

it is the value that must be realized on the wheels. In our case this slip must not be larger than 0.2 (or the value according to the maximal longitudinal force). Up to this value the slip can be increased linearly as a function of the longitudinal force.

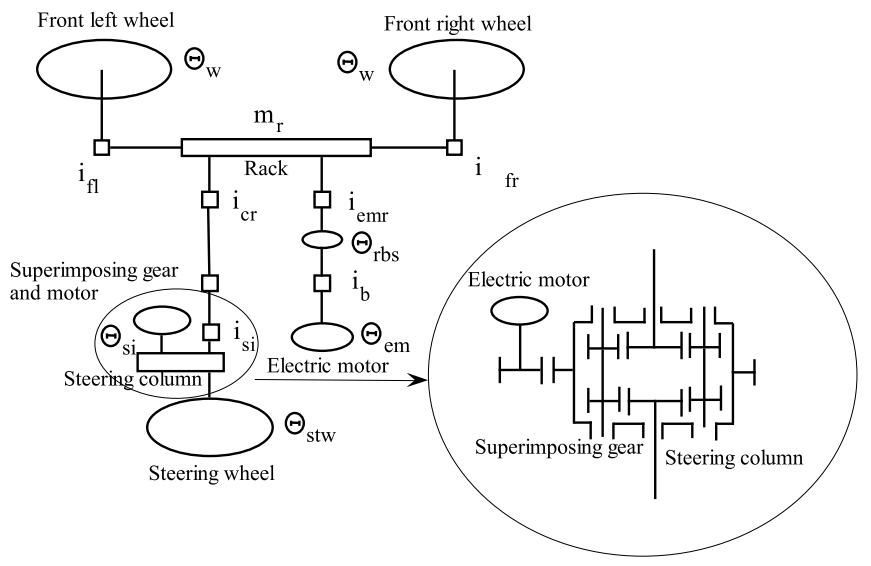

Fig. 4. Elementary model of SIA

\subsection{Suspension model}

The model of a vehicle is a five-mass (four wheels and chassis) nonlinear model. $m_{s}$ is the mass of the chassis and $m_{2 i j}$ is the unsprung mass. The position of chassis is defined by the vertical displacement of the center of gravity $\left(z_{s}\right)$, pitch angle $(\Theta)$, roll angle $(\varphi)$ and yaw angle $(\psi)$ (Fig. 3). In the dynamic equations the pitch, roll and yaw dynamics, and inertias $I_{\theta}, I_{\varphi}$, $I_{\psi}$ are considered. The force elements in suspension system are the damper forces, the sprung forces and the active suspension forces. $F_{t r}, F_{W}, \Delta F_{b i}$ are the tractive force, the side-wind force, and the brake force difference on the axle, respectively. The disturbances of model are road excitations $w_{i j}$ and wind forces. In the suspension equations $\delta_{f}$ is the front wheel steering, $v$ is the velocity, $\beta$ is side slip angle of full car model.

$l_{f}\left(l_{r}\right)$ are distances between the front (rear) axle and the car chassis at the center of gravity, $h_{C G}$ is height of center of gravity, $h_{f}$ and $h_{r}$ are distance between left (right) wheel and car chassis at center of gravity and $h_{s}$ is the arm if the roll moment. Furthermore, $k_{1 i j}, k_{2 i j}$ are stiffnesses of the suspension and the tyres, $c_{1 i j}, c_{2 i j}$ are damping coefficients of the suspension and the tyres, $K_{i}$ is the stiffness of the auxiliary anti roll bar (front/rear stabilizers) and $K_{1 i j}$ is the tyre model constant. 


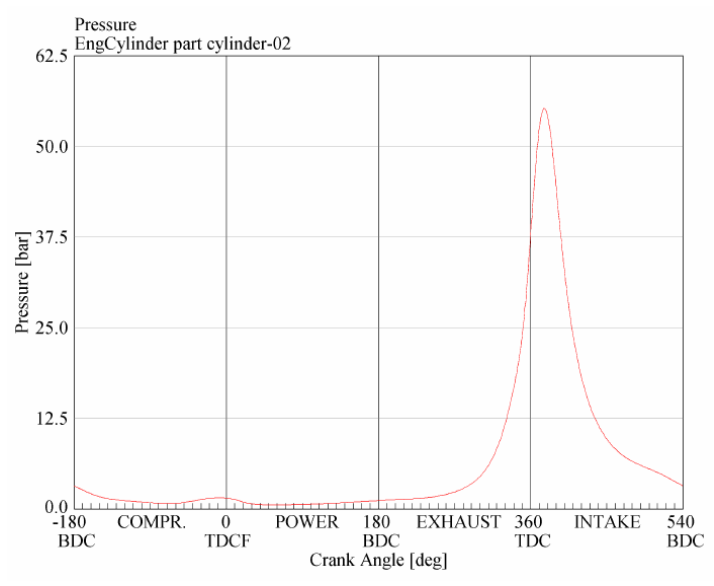

a) Cylinder pressure by GT-Power

Brake Torque, Part cranktrain-01

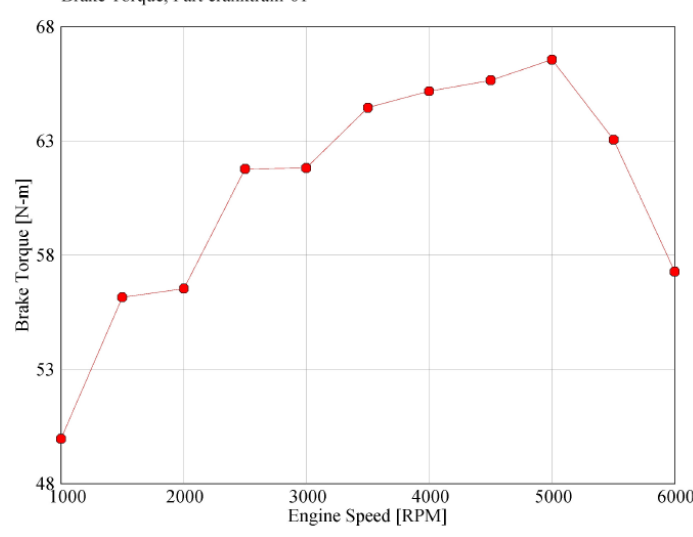

c) Engine torque by GT-Power

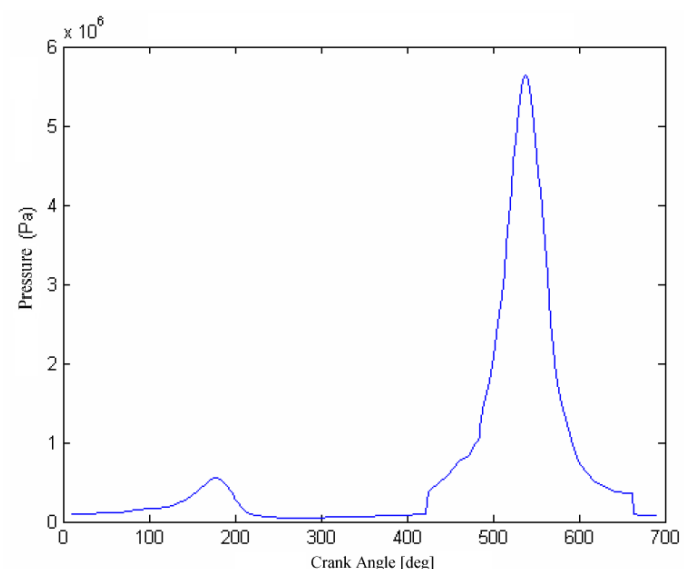

b)Cylinder pressure by Matlab

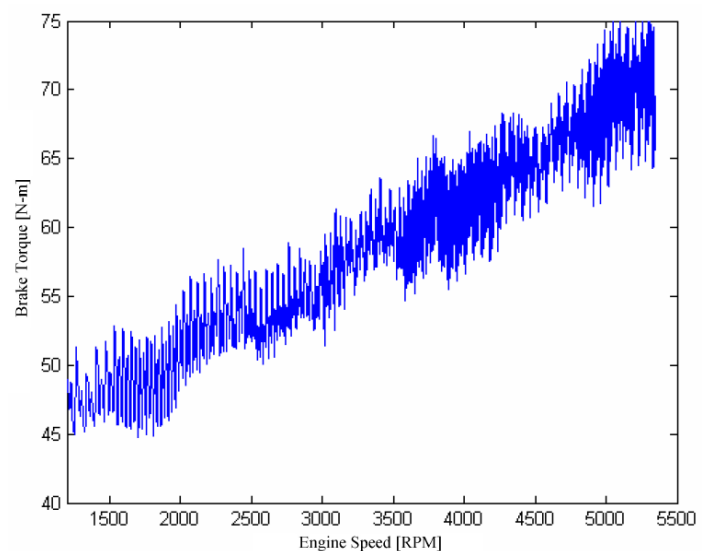

d) Engine torque by Matlab

Fig. 5. GT-Power and Matlab simulation results

Control orientated a nonlinear model is defined by the following equations [9]. First the differential equations of vehicle dynamics are formalized such as the pitch torques, roll torques, yaw torques, lateral forces, vertical forces.

$$
\begin{aligned}
& I_{\Theta} \ddot{\Theta}-l_{f}\left(F_{1 f l}+F_{1 f r}\right)+l_{r}\left(F_{1 r l}+F_{1 r r}\right)+ \\
& \quad+h_{C G} F_{t r}=0 \\
& I_{\varphi} \ddot{\varphi}-h_{s} m_{s} v(\dot{\beta}+\dot{\psi})+h_{f}\left(F_{1 f l}-F_{1 f r}\right)+ \\
& \quad+h_{r}\left(F_{1 r l}-F_{1 r r}\right)-m_{s} g h_{s} \varphi+h_{s} F_{W}=0 \\
& I_{\psi} \ddot{\psi}-l_{f}\left(S_{f l}+S_{f r}\right)+l_{r}\left(S_{r l}+S_{r r}\right)-\sum M_{i j}+ \\
& \quad+l_{l} F_{W}+l_{f} \Delta F_{b f}+l_{r} \Delta F_{b r}=0 \\
& m v(\dot{\beta}+\dot{\psi})-m_{s} h_{s} \ddot{\varphi}-\sum S_{i j}-F_{W}=0 \\
& m_{s} \ddot{z_{s}}+\sum F_{1 i j}=0
\end{aligned}
$$

Then the vertical forces are formalized on wheels and in the suspension systems:

$$
\begin{aligned}
& m_{2 i j} z_{2 i j}-F_{1 i j}+F_{2 i j}=0, \\
& F_{1 i l}= k_{1 i l}\left(z_{1 i l}-z_{2 i l}\right)+c_{1 i l}\left(z_{i i l}-z_{2 i l}\right)- \\
&-K_{i} \frac{\varphi-\left(z_{2 i l}-z_{2 i r}\right) / 2 h_{i}}{2 h_{i}}-F_{i l} \\
& F_{1 i r}=k_{1 i r}\left(z_{1 i r}-z_{2 i r}\right)+c_{1 i r}\left(z_{1 i r}-z_{2 i r}\right)- \\
&-K_{i} \frac{\varphi-\left(z_{2 i l}-z_{2 i r}\right) / 2 h_{i}}{2 h_{i}}-F_{i r}
\end{aligned}
$$

Suspensions compressions depend on the vertical displacement and its rate, and the roll and pitch of the chassis. Restoring forces on the tyre:

$$
F_{2 i j}=k_{2 i j}\left(z_{2 i j}-w_{i j}\right)+c_{2 i j}\left(z i_{2 i j}-w_{i j}\right)
$$

The cornering forces of tyres $\left(S_{i j}\right)$ and angle torques $\left(M_{i j}\right)$ could be approximated by a cubic equation with measurement constants, but for control design they are calculated as linear functions. The dynamic effects of vertical load are neglected.

$$
\begin{aligned}
S_{i j} & =\mu W_{i j}\left[\psi_{i j}-\frac{1}{3} \psi_{i j}^{2}-\frac{1}{27} \psi_{i j}^{3}\right] \\
M_{i j} & =e_{i j} \mu W_{i j}\left[\psi_{i j}-\psi^{2}+\frac{1}{3} \psi_{i j}^{3}-\frac{1}{27} \psi_{i j}^{4}\right]
\end{aligned}
$$

where: $\psi_{i j}=\frac{K_{i j}}{\mu W_{i j}} \tan \beta_{i}$. The cornering powers are:

$$
K_{i j}=K_{1 f l} W_{i j}+K_{2 i j} W_{i j}^{2}
$$




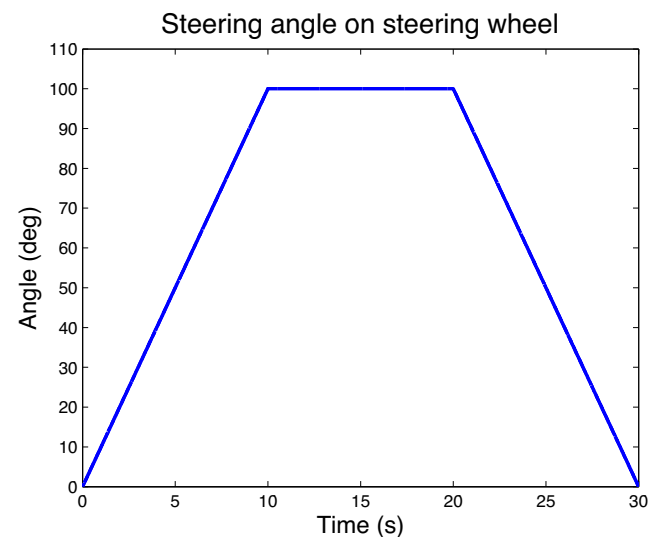

a) Steering angle

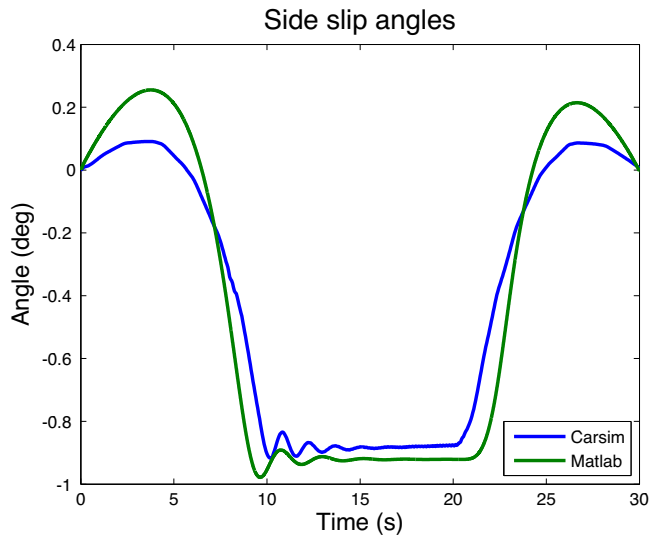

c) Side slip angle

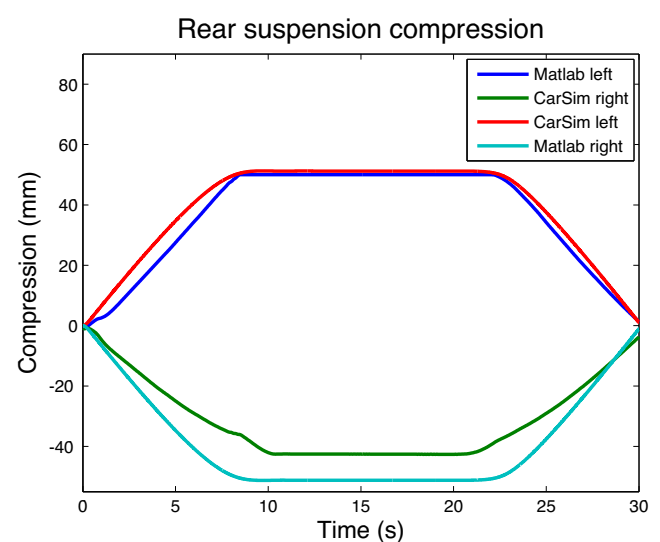

b) Rear suspension compression

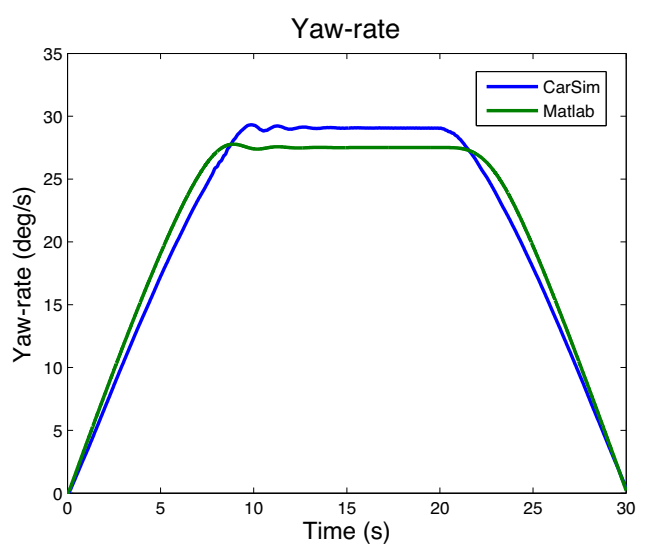

d) Yaw-rate

Fig. 6. Carsim and Matlab simulation results

where $W_{f j}=\left[m_{2 f j}+\frac{m_{s} l_{r}}{2\left(l_{f}+l_{r}\right)}\right] g-F_{2 f j}, W_{r j}=\left[m_{2 r j}+\right.$ $\left.\frac{m_{s} l_{f}}{2\left(l_{f}+l_{r}\right)}\right] g-F_{2 r j}$.

The lateral tire forces in the direction of the wheel-groundcontact velocity are approximated proportionally to the tire sideslip angle $\alpha . F_{y, f}=\mu C_{f} \alpha_{f}, F_{y, r}=\mu C_{r} \alpha_{r}$. Here $C_{i}$ is the tire side-slip constant and $\alpha_{i}$ is the tire side-slip angle associated with the front and rear axles. The chassis and the wheels have identical velocities at the wheel ground contact points. At stable driving conditions the tire side slip angles are

$$
\begin{aligned}
& \beta_{f}=-\beta+\delta_{f}-\frac{l_{f} \cdot \dot{\psi}}{v} \\
& \beta_{r}=-\beta+\frac{l_{r} \cdot \dot{\psi}}{v}
\end{aligned}
$$

It is necessary to linearize this model for control purposes. In this case suitable states for defining a linear system are chosen and the nonlinear effects are considered disturbances. By the linearization the velocity and vertical load of tyre are constant, the cornering power is linear: $K_{i j}=K_{1 f l} W_{i j}$. The axle-slip angles are relatively small, $\operatorname{sot} \tan \beta$ is approximated by constant $\beta$.

\subsection{Steering model}

The Superimposed Actuator (SIA) steering system contains mechanical connection between the steering wheel and the steered wheels, but it is able to add steering angle to the driver's original angle value by using a superimposing gear and it in- creases vehicle stability $\left( \pm 100^{\circ}\right.$ steering column angle and $\pm 8^{0}$ steering wheel angle). In these systems harmonic or epicyclic gears are used for this purpose because of their high drive ratio.

The basic model of SIA is illustrated in Fig. 4 . In SIA the steering system must be moved by the electric motor of the superimposed gear or also the driver, therefore the whole inertia must be computed. Obviously, the driver does not need to do it alone, the electric motor at rack reduces the necessary steering force required from the driver and the other motor at the superimposing gear influences the steering angle.

The model of the SIA is illustrated in Fig. $4 \theta_{w}, \theta_{r b s}, \theta_{s i}$ and $\theta_{s t w}$ are the inertias of wheels on axis of steering, the inertia of ball-spindle, and the inertia of electric motor and the inertia of steering wheel, respectively. $i_{f l}, i_{r l}, i_{c r}, i_{e m r}, i_{b}$ and $i_{s i}$ are ratios of the wheels and arms, ratio of rack-and-pinion steering gear, the ratio of the ball-spindle, the ratio of the cogged belt, and the ratio of superimposing gear, respectively.

To compute the inertia of the steering system it is necessary to reduce all of them to a predefined axis, e.g. to the steering column. The reduced inertia of the rack and arms is $\theta_{r r}=m_{r} C^{2}$, where $m_{r}$ is the mass of rack and arms, $C$ is a constant for reduction. The reduced inertia of the wheels is $\theta_{w r}=\theta_{w}\left(\frac{1}{i_{f l}}+\frac{1}{i_{f r}}\right) i_{c r}$. The reduced inertia of the power-assisted electric motor and the ball-spindle is $\theta_{m r}=\theta_{e m} \frac{i_{c r}^{2}}{i_{e m r}^{2}} i_{b}^{2}+\theta_{r b s} \frac{i_{c r}^{2}}{i_{e m r}^{2}}$. The reduced inertia of the superimposing gear and electric motor is $\theta_{s i r}=\theta_{s i} \frac{1}{i_{s i}}$ The 
inertia of the whole system reduced to the steering column is the sum of all of them:

$$
\theta_{d}=\theta_{r r}+\theta_{m r}+\theta_{w r}+\theta_{s i r}+\theta_{s t w}
$$

\section{Simulation results}

In simulations the previously described and Matlab-encoded models are validated. The non-linear engine model is validated using GT-Power, the simulation results of the global vehicle model are compared with CarSim. Figs. 5 shows the most important characteristics of the engine computed by Matlab and GT-Power. The results of Matlab model up to 5000 1/min rev approximate well the results of the more complex GT-Power, therefore it can be used to check the designed controller. At higher rev engines the approximation error increases. The reason for the error is the imperfection of the applied intake manifold model. The difference of crankshaft angles comes from the difference in the top dead center position in the crankshaft angle in the Matlab and in the GT-Power model. In case of the Matlab model the crankshaft positions are $180^{\circ}$ earlier.

In the CarSim simulation a steering maneuver is applied. The steering wheel rotation is shown in Fig. 6.a). This input causes the cornering of the vehicle, whose effect is a roll motion and suspension compression, see Fig. 6.b). During cornering a sideslip arises on the vehicle, and the yaw-rate value also increases. In all simulation cases the results of the Matlab model approximate the CarSim results well. The reason for the differences is the neglected dynamic effects in the Matlab model.

\section{Conclusion}

In this paper four vehicle components have been formalized. In order to be used for control-oriented modeling all non-linear models are linearized. In the formalized models the actuators' effects and electronic input possibilities are shown. The constructed vehicle model can be used for model-based integrated control design. High-complexity programs are used for the validation of vehicle models, which are encoded in Matlab/Simulink. The engine-model is analyzed using GT Power, the behaviour of the global vehicle is verified by CarSim. These simulations show that the formalized models approximate well the more complex models, therefore they can be used for further control design and analysis.

\section{References}

1 Alvarez L, Horowitz R, Safe platooning in automated highway systems, Vehicle System Dynamics, 1999, pp. 23-84, DOI 10.1076/vesd.32.1.23.4228, (to appear in print).

2 Dezsényi Gy, Emőd I, Finichiu L, Design and analysis of internal combustion engines, Nemzeti Tankönyvkiadó, Budapest, 1999. in Hungarian.

3 Gillespie TD, Fundamentals of vehicle dynamics, Society of Automotive Engineers Inc., Warrendale, 1992.

4 Kalmár I, Stukovszky Zs, Processes of internal combustion engines, Múegyetemi Kiadó, Budapest, 1998. in Hungarian.

5 Kiencke U, Nielsen L, Automotive control systems for engine, driveline and vehicle, Springer, Berlin, 2000.
6 Kuti I, Szőke D, Three dimensional semi-rigid ring tyre model and its application to the transient analysis of vehicles, 16th IAVSD Symposium (Pretoria, South Africa), 1999.

7 Pacejka HB, Tyre and vehicle dynamics, Butterworth-Heinemann, 2006.

8 Souder JS, Powertrain modeling and nonlinear fuel control, 1998. MSc Thesis.

9 Yoshimura Y, Emoto Y, Steering and suspension system of a full car model using fuzzy reasoning based on single input rule modules, International Journal Vehicle Autonomous Systems, posted on 2003, DOI 10.1504/IJVAS.2003.003537, (to appear in print). 